\title{
Masson's Hemangioma of the Cheek: A Case Report
}

\author{
K. K. Shrestha ${ }^{1}$ A. K. Jha ${ }^{1}$ - R. R. Joshi ${ }^{1}$ A. S. Rijal ${ }^{1}$ - A. Dhungana ${ }^{1}$. \\ S. Maharjan ${ }^{1}$
}

Received: 21 April 2015/ Accepted: 29 July 2015/Published online: 31 July 2015

(C) Association of Otolaryngologists of India 2015

\begin{abstract}
We report a rare case of intravascular papillary endothelial hyperplasia (IPEH) of the cheek. This neoplasm, known as Masson's tumor, is an unusual vascular lesion of proliferating endothelial cells. It is usually confined to the lumen of preexisting vessels or vascular malformations. The principal significance of IPEH is its resemblance to a variety of benign and malignant diseases and possible misdiagnosis as such. Achieving a correct diagnosis is essential to avoid subjecting a patient to either unnecessarily aggressive or inadequate therapy. For this reason, awareness of this lesion is very important.
\end{abstract}

Keywords Intravascular endothelial hyperplasia . Masson's tumor · Cheek

\section{Introduction}

Intravascular papillary endothelial hyperplasia (IPEH) is an unusual benign vascular lesion comprising approximately $2 \%$ of the vascular tumors of the skin and subcutaneous tissue [1]. It was first described by Masson [2] in 1923 as a 'hemangioendotheliome vegetant intravasculaire', and has subsequently been described by a variety of names. The term IPEH is the most descriptive and least confusing and is the one most frequently used in the English literature [3].

K. K. Shrestha

kundanshrestha9@hotmail.com

1 Department of Otolaryngology \& Head and Neck Surgery, Nepal Medical College Teaching Hospital, P O Box 13344, Attarkhel, Jorpati, Kathmandu, Nepal
We present a case of a Masson's hemangioma that came to ENT and HNS outpatient department (OPD) of Nepal Medical College Teaching Hospital (NMCTH).

\section{Case Report}

A 16 year old girl from Sindhupalchowk came to ENT OPD of NMCTH with swelling in the right cheek for 6 years. It was insidious in onset and gradually progressive. There was no pain to begin with but for the past 3 years, she complained of mild pain on and off with no radiation and no aggravating and relieving factors. She also gave history of intraoral incision and drainage 3 years back from the same site following which the swelling had subsided only to recur after 2 months.

On examination, the right cheek showed diffuse fullness below the maxillary prominence. Intra oral examination revealed a $3 \times 2 \mathrm{~cm}$ soft, boughy swelling in right buccal mucosa around $2 \mathrm{~cm}$ behind the oral commissure. There was a linear faint scar over the swelling around $2 \mathrm{cms}$ in anteroposterior direction. It was non tender, non fluctuant, non reducible and non compressible on palpation. Other ENT examinations were normal.

The patient was worked up in NMCTH. FNAC from right masseter mass came out to be malignant mesenchymal neoplasm. Slide review done outside gave same result and advised biopsy for more detail typing and confirmation. Plain and contrast computerized tomographic scan was done which showed hypertrophied right masseter muscle containing heterogeneously enhancing necrotic mass approximately $40 \times 30 \times 25 \mathrm{~mm}$ in anteromedial aspect with multiple feeder vessels. There was no evidence of bony destruction. Medially the mass was abutting the lateral pterygoid muscle without local invasion (Figs. 1,2). 
Patient underwent excision under general anaesthesia through intra oral approach. A $2 \mathrm{~cm}$ linear incision was made over the previous scar and flaps raised on either side. The mass was gently dissected, excised in toto and sent for histopathological examination.

Microscopic sections (Figs. 3, 4) showed dilated blood vessel with multiple, small, delicate papillary structures projecting into the lumen. These papillae were lined by single layer of plump endothelial cells with a hyalinized core. Some of the lumen showed thrombi. No evidence of increased mitotic activity was observed and no atypia of the endothelial lining was evident. A final diagnosis of IPEH/Masson's tumor was made.

\section{Discussion}

Three different types of IPEH have been reported: (a) a primary (pure) form where changes are observed in a distended vessel; (b) a secondary (mixed) form that occurs in preexisting varices, hemangiomas, pyogenic granulomas, or lymphangiomas; and (c) an uncommon type in an extravascular location [1].

Although the most common sites of IPEH are head and neck, fingers and trunk, these tumors may occur in any blood vessel. However, occurrence of IPEH in the oral cavity is extremely rare. A review in the accessible literature showed less than 80 cases of IPEH in the oral mucosa and lips.
Fig. 1 CT scan of the patient (Coronal cuts)

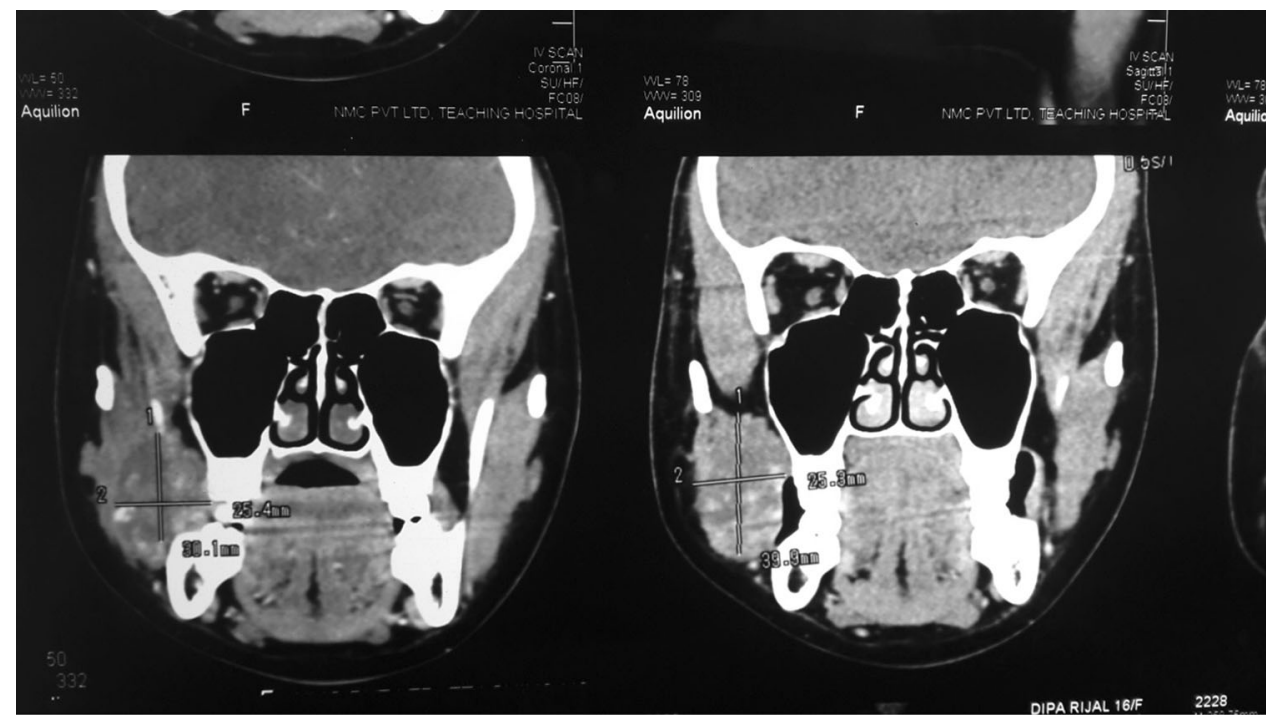

Fig. 2 CT scan of the patient (Axial cuts)

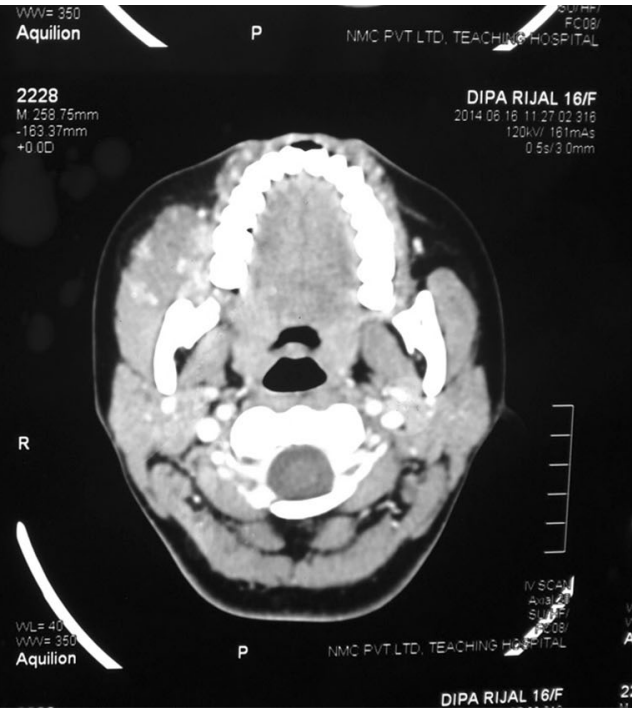


Fig. 3 Histopathological slide of the specimen (low power)

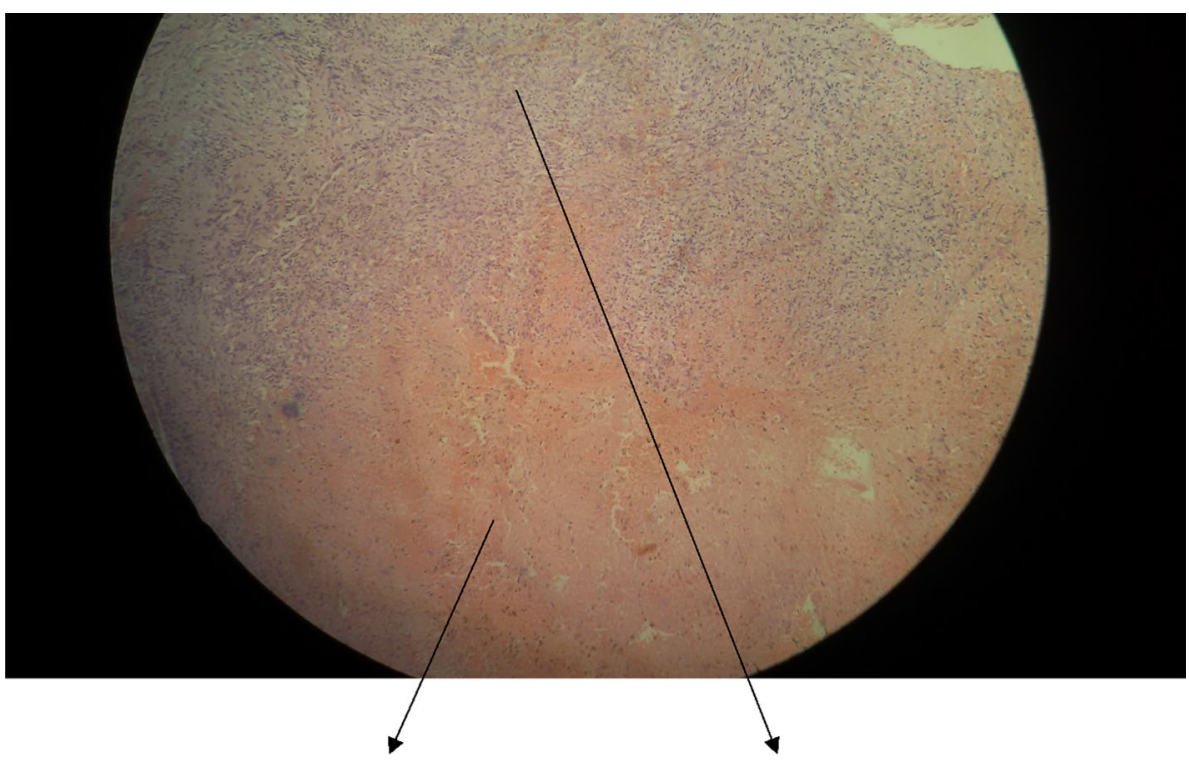

Pink central area of blood thrombosis

Peripheral endothelial proliferation

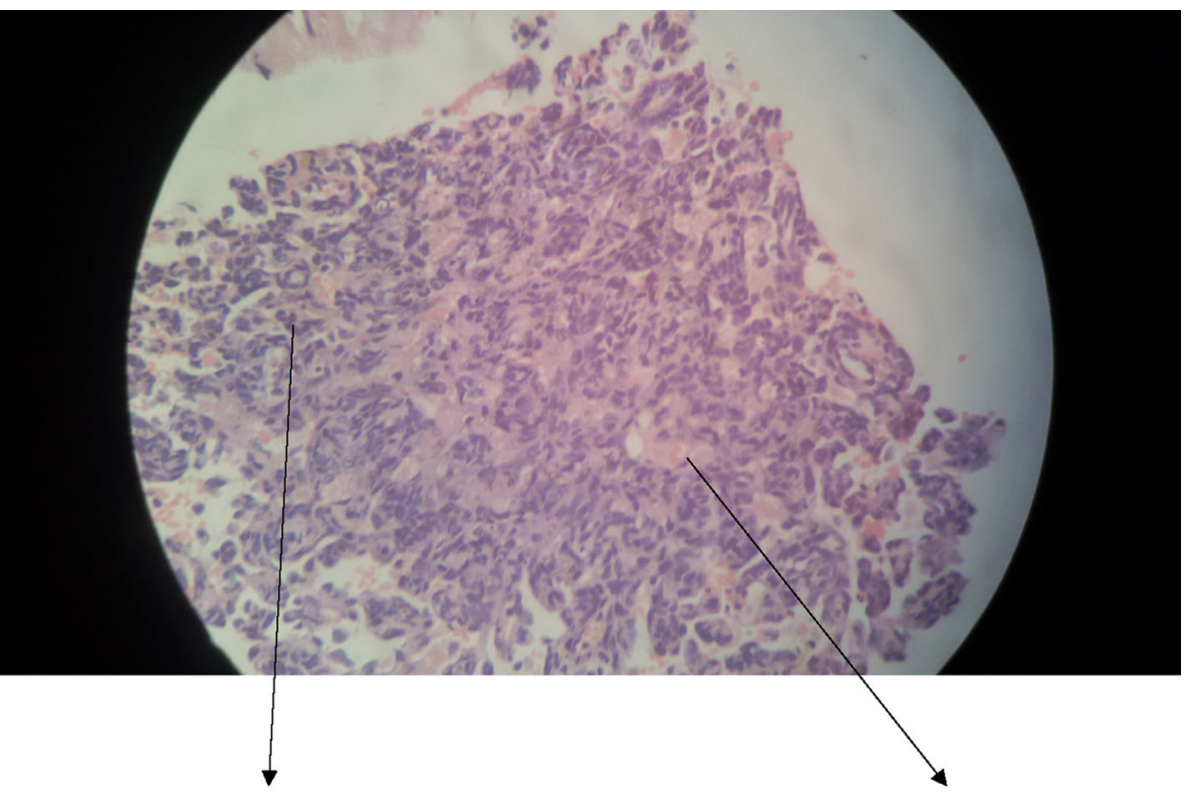

Endothelial proliferation with papillary projections

Capillaries with blood cells inside
IPEH of the oral mucosa and lips occurs more commonly in females than males. A possible hormonal role has been suggested based on this gender difference, and local angiogenic growth factors may contribute to endothelial proliferation [3]. Our case was no different.

In the oral cavity, IPEH presents as a slow-growing, firm, reddish-blue mass with slight elevation. The most frequent locations are the lower lip, tongue, buccal mucosa, upper lip, mandibular vestibule and angle of the mouth [3]. The presentation of our patient was similar.

The lesion has been clinically mistaken for mucocele, hemangioma, lymphagioma, hematoma, Kaposi sarcoma, hemangioendothelioma, thrombosed vein, traumatic fibroma, pyogenic granuloma, angiosarcoma and salivary gland tumor [3]. In our case, we had an earlier opinion of lymphangioma.

Although benign, this lesion is clinically important because it presents as a mass lesion that may be mistaken histologically for angiosarcoma, and it tends to recur if incompletely resected [4, 5]. Correct diagnosis of the lesion is essential to prevent overly aggressive treatment.

The pathogenesis of IPEH is poorly understood. One possible mechanism is a benign neoplastic process involving endothelial cell proliferation and papillary formation in the vascular lumen that undergoes degeneration 
and necrosis in the manner of a red infarct. Alternative mechanisms include a benign endothelial proliferation arising from a thrombus as a variant of angiolymphoid hyperplasia with eosinophilia; a reactive process of endothelial cells induced by blood stasis and perivascular inflammation; and a pseudotumoral lesion caused by endothelial proliferation with papillary formation proceeded by an accumulation of thrombotic material, which serves to facilitate development of the lesion [6].

The benign behavior of these lesions is emphasized throughout the literature. The vast majority of lesions present as a slowly growing mass that can be cured by local excision $[4,6]$.

The best treatment is a total excision-biopsy with healthy margins. When resected completely, recurrence is extremely rare.

\section{Conclusion}

The importance of this entity is its ability to mimic a variety of diseases both benign and malignant in the orofacial region. Awareness of this lesion will prevent incorrect diagnoses and overly aggressive treatment. Thus, it is very important for ENT and Head and Neck surgeons to recognize this lesion.

\section{References}

1. Tosios K, Koutlas IG, Papanikolaou SI (1994) Intravascular papillary hyperplasia of the oral soft tissues: report of 18 cases and review of the literature. J Oral Maxillofac Surg 52:1263-1268

2. Masson M (1923) Hemangioendotheliome vegetant intra-vasculaire. Bull Soc Anat Paris 93:517-523

3. Makos CP, Nikolaidou AJ (2004) Intravascular papillary endothelial hyperplasia (Masson's tumor) of the oral mucosa. Presentation of two cases and review. Oral Oncol Extra 40:59-62

4. Avellino AM, Grant GA, Harris AB (1999) Recurrent intracranial Masson's vegetant intravascular hemangioendothelioma. J Neurosurg 91:308-312

5. Salyer WR, Salyer DC (1975) Intravascular angiomatosis: development and distinction from angiosarcoma. Cancer 36:995-1001

6. De Courten A, Keuffer R, Samson J, Lombardi T (1999) Intravascular papillary endothelial hyperplasia of the mouth: report of six cases and literature review. Oral Dis 5:175-178 